\title{
A novel geo-statistical approach for transport infrastructures network monitoring by Persistent Scatterers Interferometry (PSI)
}

\author{
Valerio Gagliardi \\ Department of Engineering \\ Roma Tre University \\ Via Vito Volterra 62, Rome, Italy \\ valerio.gagliardi@uniroma3.it
}

\section{Fabio Tosti}

School of Computing and Engineering University of West London (UWL)

St Mary's Road, Ealing, London, UK Fabio.Tosti@uwl.ac.uk

\author{
Luca Bianchini Ciampoli \\ Department of Engineering \\ Roma Tre University \\ Via Vito Volterra 62, Rome, Italy \\ luca.bianchiniciampoli@uniroma3.it
}

\author{
Amir M. Alani \\ School of Computing and Engineering \\ University of West London (UWL) \\ St Mary's Road, Ealing, London, UK \\ Amir.Alani@uwl.ac.uk
}

\author{
Fabrizio D'Amico \\ Department of Engineering \\ Roma Tre University \\ Via Vito Volterra 62, Rome, Italy \\ fabrizio.damico@uniroma3.it
Andrea Benedetto
Department of Engineering
Roma Tre University
Via Vito Volterra 62, Rome, Italy
andrea.benedetto@uniroma3.it

\begin{abstract}
Persistent Scatterer Interferometry (PSI) is an Interferometric Synthetic Aperture Radar (InSAR) technique based on a multi-temporal interferogram analysis of SAR images. The aim of the technique is to extract long-term high phase stability benchmarks of coherent point targets, namely Persistent Scatterers (PS). In the last decades, several approaches have been developed to obtain PSI point targets, proving their viability for applications to transport infrastructure monitoring and surveillance. However, SAR satellites can only detect displacements in the Line-of-Sight (LoS), with reference to the specific orbit-related incident angle. This work proposes a novel geo-statistical approach to ease postprocessing of large datasets of PSs resulting from the application of the PSI algorithms over an area of interest. The approach aims at correcting the component of the displacement collected from the acquisition geometry of the sensor.
\end{abstract}

Keywords-Persistent Scatterers Interferometry (PSI), Transport Infrastructure Maintenance, Acquisition geometry, geo-statistical approach, Line of Sight (LoS)Introduction (Heading 1)

\section{INTRODUCTION}

Persistent Scatterer Interferometry (PSI) is an Interferometric Synthetic Aperture Radar (InSAR) technique based on a multi-temporal interferogram analysis of SAR images, use to extract long-term high phase stability benchmarks of coherent PS point targets, namely Permanent Scatterers (PS) [1].

A major advantage of the PSI techniques is that SAR data can be acquired in daytime and nighttime, since they are independent from weather conditions and variations in lighting conditions. This is different from optical satellite sensors, that strictly require sunlight for data collection purposes. As a result of this, new datasets are available at each satellite orbit overpassing the same area. In the past few years, several approaches have been developed to obtain these PSI point targets. Among the others, PSInSAR ${ }^{\mathrm{TM}}[1,2,3]$, the SqueeSAR [4] and Small Baseline Subset (SBAS [5, 6]) techniques, have proven to work effectively for transport infrastructure monitoring and surveillance. However, SAR satellites can only detect displacements in the Line-of-Sight, with reference to the specific orbit-related incident angle. This work aims at provide a novel geo-statistical approach to efficiently post-process large amounts of PSs resulting from the application of the PSI algorithms over an area of interest, in order to correct the component of the displacement deriving from the acquisition geometry of the sensor.

\section{PSI FOR TRANSPORT INFRASTRUCTURE MONITORING}

The working framework of the PSI techniques relies on a statistical analysis of the signals emitted by the on-satellite sensor and back-scattered by a network of coherent targets on the ground, i.e., the Permanent Scatterers (PS). This approach allows to estimate the displacements occurred between different acquisitions by separation between the phase shift related to the ground motions and the phase component due to the atmosphere, the topography and the signal noise contributions $[1,7]$.

An advantage of these techniques is the relatively lighter data-processing required for the assessment of displacements and the detection of critical areas, as opposed to the higher computational load needed in other approaches [1,2]. As a consequence, the PSI technique sets as an ideal technique in monitoring transport infrastructure, since the high density of radar stable targets allows for highly accurate measurements. This is more evident in rural environment where roads, railways and runways can typically generate numerous PSs. Several applications of PSI related to the transport infrastructure monitoring can be found in the literature, such as [8-15], confirming its applicability to this area of endeavour.

Nowadays, various different in-situ technologies for subsidence and displacement mapping are available (e.g., GPS [16], levelling [17], strain gauges [18], laser scanner, [19]). However, their application is generally resource-consuming, as it often requires closure of the infrastructure to traffic and the presence of experienced surveyors on-site. The use of satellite SAR imagery allows to overcome these limitations, as measurements over large areas and, under suitable conditions, spatially dense information on slow groundsurface deformations are possible. In addition, the PSI technique provides a high accuracy, ranging from 1 to $3 \mathrm{~mm}$, on single measurements for each SAR acquisition in regard to the Line-of-Sight (LOS) deformation rate [1].

\section{PROBLEM STATEMENT}

The acquisition of SAR satellite-images occurs along two different polar-orbits, respectively referred to as Ascending, 
i.e. if the satellite moves in a South-North direction, and Descending, i.e., if the satellite moves in a North-South direction. According to the acquisition orbit (i.e. the geometry), the Displacements are measured along a unit vector co-directional to the satellite, named LOS. Based on the specific orbit and position of the area of interest, different incidence angles of the signal on the ground can be observed. (Fig.1)
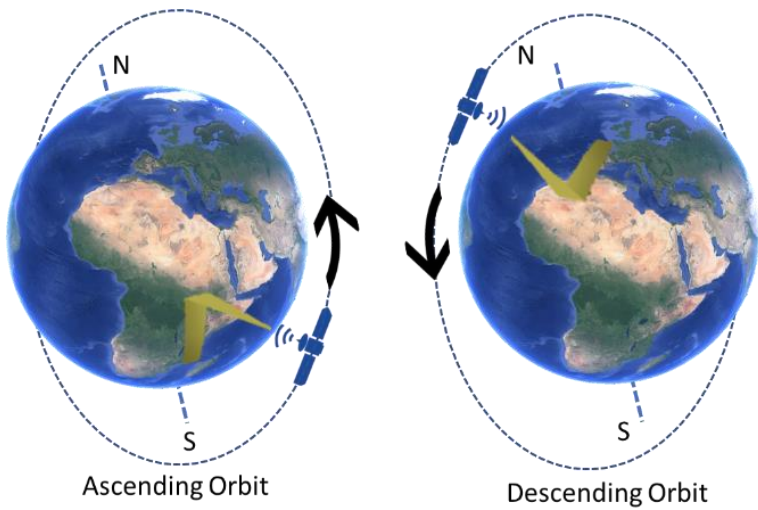

Fig.1 SAR data acquisition along an Ascending and Descending orbit

It is worth noting that the displacements detected in correspondence of a PS are 1D measurements. This involves that displacement values are defined as the projection of the $3 \mathrm{D}$ component of the actual displacement vector on the satellite LOS direction.

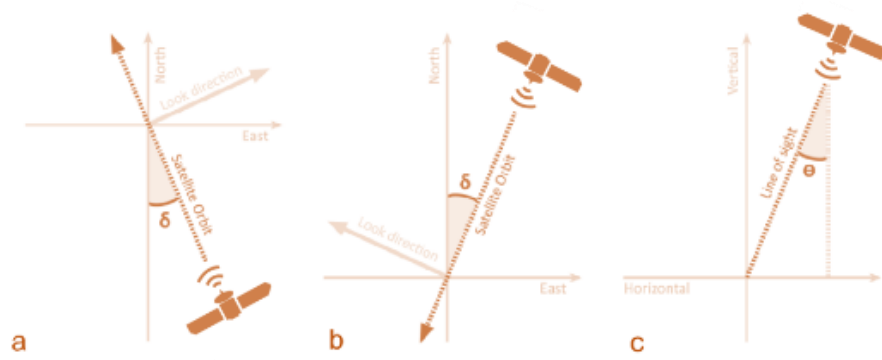

Fig. 2. Satellite acquisition geometry: look direction and angle between the azimuth and the north direction, $\delta$, for (a) ascending and (b) descending modes; (c) local incidence angle, $\theta$.

As a consequence, no-phase variation is detected in case the radar target is affected by a displacement along the direction perpendicular to the LOS. Furthermore, it should be noted that no motion components can be detected by the SAR systems along the North-South direction. This is due to the fact that these components do not create a significant range variation, due to the satellite orbits almost parallel to the Earth meridians.

The use of a single SAR image in an acquisition geometry does not allow to discriminate between horizontal or vertical movements, as it only defines the displacement on the LoS direction. However, if PS data are available for both Ascending and Descending acquisition geometries, it is possible to estimate the two components of the real displacement vector. To this effect, a specific processing algorithm is presented in the section IV of this paper.

\section{Methodology}

Let us consider a Cartesian reference system (x, y, z), where the three reference directions are parallel to the Easting (x), the Northing (y), and the vertical direction(z), respectively. If the PS, referred to the same structure, are identified in both descending and ascending data-sets, the PS technique can provide two components of the 3D displacement vector affecting this radar target. The PS average velocity-vector can be written as the following equation:

$$
\left[\overrightarrow{\boldsymbol{V}}_{\text {real }}\right]=\left[\begin{array}{lll}
\overrightarrow{s_{x}} & \overrightarrow{s_{y}} & \overrightarrow{s_{z}}
\end{array}\right]\left[\begin{array}{c}
\mathrm{V}_{\mathrm{E}} \\
\mathrm{V}_{\mathrm{v}} \\
\mathrm{V}_{\mathrm{N}}
\end{array}\right]
$$

where $V_{V}, V_{E}$, and $V_{N}$ are the components of the velocity vector along vertical, East-West, and North-South direction respectively, and $\overrightarrow{S_{x}} \overrightarrow{S_{y}} \overrightarrow{S_{z}}$ are the versors of the Cartesian system.

In Fig. 3, the Vertical $\left(\mathrm{V}_{\mathrm{V}}\right)$ and Horizontal $\left(\mathrm{V}_{\mathrm{E}}\right)$ components in East-West direction of the real-velocity vector $\left(\mathbf{V}_{\text {real }}\right)$ is obtained by projecting the Ascending $\left(\mathrm{V}_{\mathrm{A}}\right)$ and Descending $\left(V_{D}\right)$ velocity values in the LoS direction, referred to the relevant acquisition geometries.

Since both acquisition geometries are known, the following system of linear equation should be considered:

$$
\left[\begin{array}{l}
V_{A} \\
V_{D}
\end{array}\right]=\left[\begin{array}{ccc}
s_{x_{-} \text {asc }} & s_{y_{-} \text {asc }} & S_{z_{-} \text {asc }} \\
s_{x_{-} \text {desc }} & s_{y_{-} \text {desc }} & S_{z_{-} \text {desc }}
\end{array}\right]\left[\begin{array}{c}
\mathrm{V}_{\mathrm{v}} \\
\mathrm{V}_{\mathrm{E}} \\
\mathrm{V}_{\mathrm{N}}
\end{array}\right]
$$

where $S_{x \_a s c}, S_{y \_a s c}, S_{z \_a s c}, S_{x \_d e s c e}, S_{y \_d e s c}, s_{z \_d e s c}$ are the direction cosines of the acquisition geometries (ascending and descending) and $\mathrm{V}_{\mathrm{A}}$ and $\mathrm{V}_{\mathrm{D}}$ are the projections of the velocity vectors along the two opposite lines of sight. Subsequently the number of unknowns $\left(\mathrm{V}_{\mathrm{V}}, \mathrm{V}_{\mathrm{E}}\right.$ and $\left.\mathrm{V}_{\mathrm{N}}\right)$ in the system is greater than the number of equations, the full 3$\mathrm{D}$ vector cannot be estimated.

However, the SAR radar is not sensitive to PS displacements in the N-S direction, since the satellite orbits are almost parallel to the Earth meridians. It is then a rational approximation to assume this component equal to zero and estimate the Easting and Vertical displacement components. Under the assumption of zero-displacements in the N-S direction, the satellite pointing parameters (i.e., the direction cosines), related to the incidence angle of the SAR sensor at the time of the acquisition, are known. 


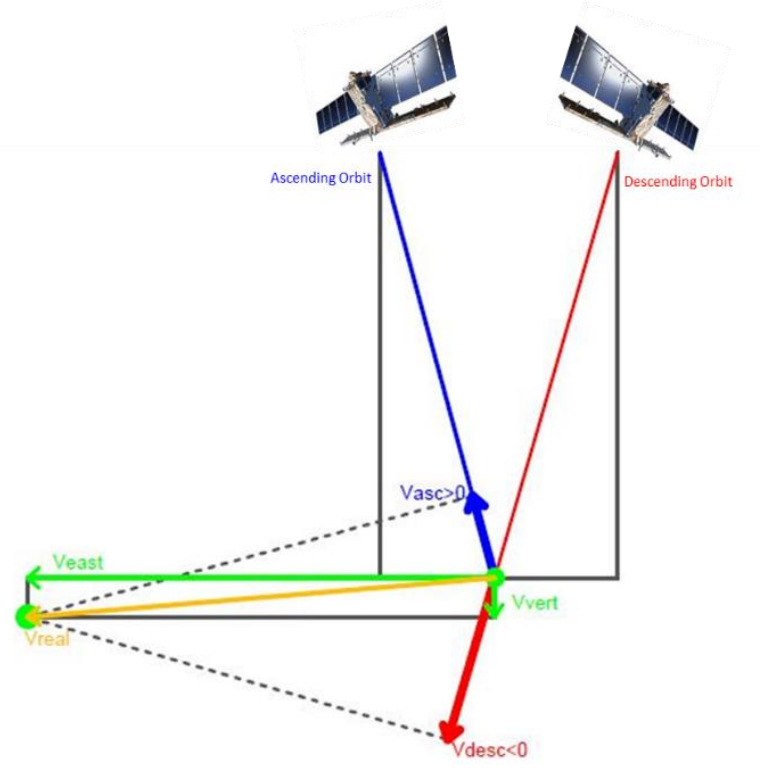

Fig. 3. Satellite acquisition geometry

If data acquired in both the acquisition geometries (Ascending and Descending) are available, this problem can be solved and the modulus of the average displacements velocity-vector, referring to the same PS radar target related to the horizontal $\left(\mathrm{V}_{\mathrm{E})}\right.$ and the vertical deformations $(\mathrm{Vv})$, can be calculated by solving the following system of equations [20,21]:

$$
\left[\begin{array}{l}
V_{A} \\
V_{D}
\end{array}\right]=\left[\begin{array}{ll}
\cos \theta_{a} & \sin \theta_{a} \\
\cos \theta_{d} & \sin \theta_{d}
\end{array}\right]\left[\begin{array}{l}
\mathrm{V}_{\mathrm{v}} \\
\mathrm{V}_{\mathrm{E}}
\end{array}\right]
$$

- $V_{A}$ and $V_{D}$ represent the velocity of deformation in Ascending and in Descending orbit, respectively;

- $\quad \mathrm{V}_{\mathrm{V}}$ and $\mathrm{V}_{\mathrm{E}}$ are the unknowns to be determined. These are the velocity in the vertical and horizontal (E-W) planes, respectively;

- $\theta_{\mathrm{a}}$ and $\theta_{\mathrm{d}}$ are the incident angles (depending on the SAR mission) in Ascending and Descending orbit, respectively (Figure 2).

The solution of this system requires every single measurement point to be recognized as a valid PS target from the satellite in both Ascending and Descending orbits, which is mostly unlikely to verify in reality.

Within this framework, an innovative geo-statistical methodology is implemented to create a regular PS-grid out of the single acquisition geometry, with a fixed spacing in each direction. The grid generates a couple of Synthetic PS by a geo-statistical interpolation of the displacement values from Ascending and Descending acquisitions. To this effect, from now on the grid will be named as Synthetic-Interpolated Grid of Persistent Scatterers (SIG-PS). In particular, the geostatistical ordinary Kriging method was used and implemented for this purpose. [22,23]

Kriging is one of the more flexible and accurate gridding methods due to the possibility to provide a representative map for different types of data-sets. It also can compensate for clustered data by giving less weight to the cluster in the overall prediction. Each grid node value is based on the known data-points neighbouring the node. Each data point is weighted by its distance away from the node and, consequently, points that are further from the node will be weighted less in the estimation of the node. To compute the $\mathrm{Z}$ value at a randomly given grid node $\mathrm{A}$, the following equation is used [22,23]:

$$
Z_{A}=\sum_{i=1}^{n} W_{i} Z_{i}
$$

Where $Z_{A}$ is the estimated value of grid node $A, n$ is the number of neighbouring data values used in the estimation, $\mathrm{Z}_{\mathrm{i}}$ is the value at location $i$ with weight, $\mathrm{W}_{\mathrm{i}}$. The value of weights will sum up to 1 to ensure that no bias is verified towards clustered data points. The weights are intended to summarise two important procedures in a spatial inference process: i) reflect the structural-proximity of samples to the estimation location; ii) -on parallel, they should have a desegregation effect, in order to avoid bias caused by potential sample clusters.

Therefore, the proposed SIG-PS method allows to define for a couple of synthetic PS having the same coordinates for point of the grid, in order to solve the system of equations reported in Eq. (1) and to combine the information related to the orbit of acquisition. As a result of this, it is possible to perform a correction of the displacements measured in the LoS direction, in order to calculate the real vector displacement-Velocity $\left(\mathrm{V}_{\mathrm{r}}\right)$.

To elaborate, the SIG-PS was applied by adopting the following protocol (Fig. 4):

I. A regular grid is created for each acquisition geometry by using the Geo-statistical Kriging Tool with the Surfer Software;

II. at every point of the grid, the centroid value is interpolated from the targets included within a circle of radius equal to $10 \mathrm{~m}$;

III. a built-in Matlab code is used to solve the system of equations in (1),

IV. as a result, the vertical $\left(\mathrm{V}_{\mathrm{V}}\right)$, and horizontal $\left(\mathrm{V}_{\mathrm{E}}\right)$ components of the actual displacement velocity vector can be obtained and represented by the same regular grid.

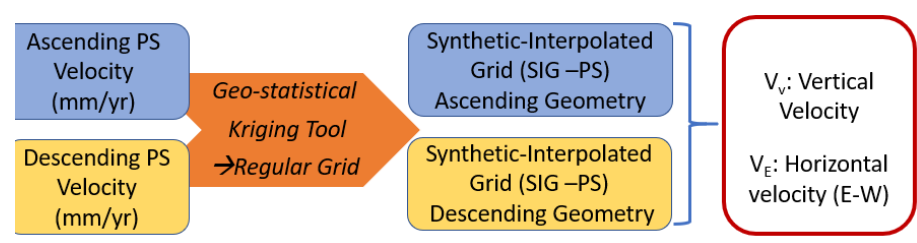

Fig. 4: Flow chart of the SIG-PS procedure used to calculate the components of the displacement velocity vector.

\section{CASE STUdy}

The methodology has been applied and tested over a airfield runway located in Italy, where the PSI data were acquired by the Sentinel 1 mission ("Data provided by the ESA: European Space Agency") in the period from 2015 to 2019. The SAR imagery included both ascending and descending orbits, with 
47 Ascending and 49 Descending Single Look Complex (SLC)-Sentinel 1A SAR Products.

\begin{tabular}{cc} 
Sentinel 1A -European Space Agency (ESA) \\
\hline Number of Images & 47 Asc - 49 Desc \\
Reference Period & $04 / 2015-01 / 2019$ \\
Frequency & $5.4 \mathrm{GHz}$ \\
Ground-Range Resolution & $5 \mathrm{~m}$ \\
Azimuth Resolution & $20 \mathrm{~m}$ \\
\hline
\end{tabular}

The PSI data have been processed following the PSInSAR $(1,2)$ approach, using the Interferometric Stacking Module of the Software SARscape [24] integrated in the Software Envi, licensed within the framework of the ESA (European Space Agency)-approved project MOBI: "Monitoring Bridges and Infrastructures Networks" (EOhops proposal ID 52479).

The results are reported in Fig. 5. The original set of PSs resulting from the processing of a single acquisition geometry data is shown in Fig.5a, whereas the result of the application of the SIG-PS method is reported in Fig. 5b. In this specific case, a $20 \times 20 \mathrm{~m}$ regular grid was adopted. In Fig. 5b, the results of the ground-truth survey (i.e. levelling by means of a total station, represented by the larger dots in the figure) are reported together with the PS analysis, proving that the method works generally well.

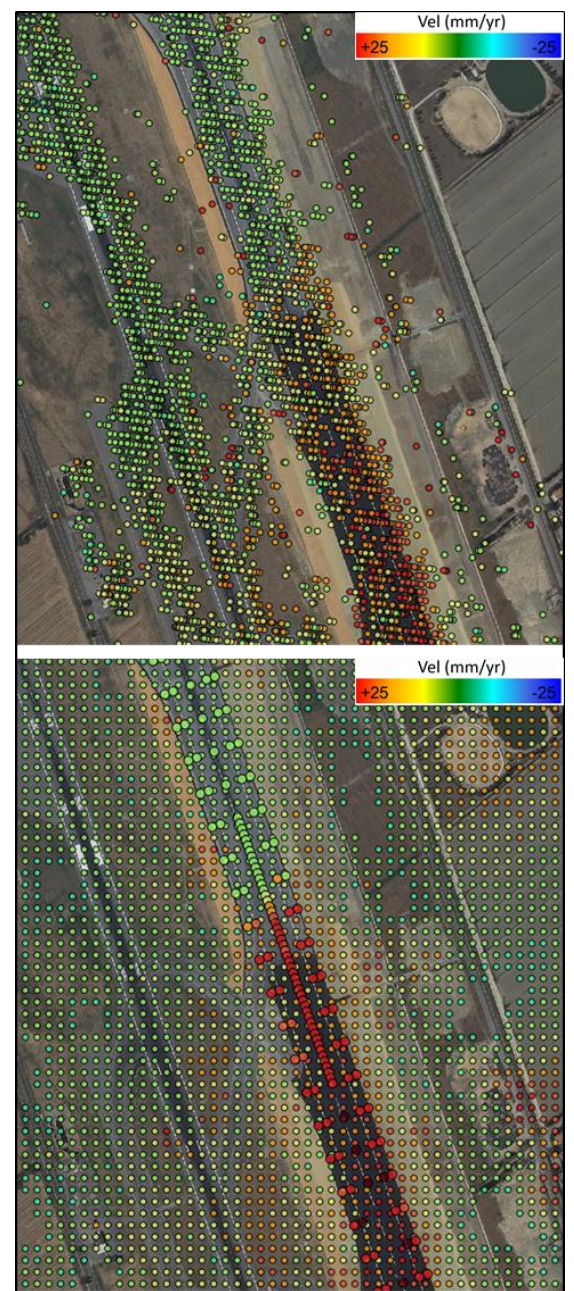

Fig 5 (a) processing of a single acquisition geometry data, (b) application of SIG-PS method
It is worth noting that defining the grid size is a crucial task for making the algorithm effective. A low amount of points within each cell should be avoided in order to ensure the statistical significance of the dataset. On the other hand, widening the cell size involves to consider PSs quite distant each to one another, thereby increasing the likelihood to disregard some subsidence effects. As a consequence, using a larger grid mesh could lead to merging displacements that should potentially be considered as independent from a geological point of view (e.g., subsidence affecting two different river basins could be mapped as a unique occurrence).

\section{CONCLUSION}

This work presents an innovative geo-statistical methodology for the calculation of the vertical and horizontal components of the displacement velocity within a PSI analysis. The methodology, named as Synthetic-Interpolated Grid of Persistent Scatterers SIG-PS, is based on the development of a regular synthetic interpolated PS grid out of the single acquisition geometry data-base. This allows to pair interpolated values of displacement velocity having the same geographical coordinates but relative to different acquisition orbits.

As a result, it is possible to solve the theoretical problem discussed in Section IV, in order to calculate the real displacement velocity vector in vertical $\left(\mathrm{V}_{\mathrm{V}}\right)$ and $\mathrm{E}-\mathrm{W}\left(\mathrm{V}_{\mathrm{E}}\right)$ directions, starting from the two vectors observed in the LoS direction of the two acquisition geometries $\left(\mathrm{V}_{\mathrm{A}}, \mathrm{V}_{\mathrm{D}}\right)$. Once the vertical and horizontal components of the deformation vector are known (positive velocities mean uplift or eastward deformations; negative velocities mean subsidence or westward deformations), it is possible to calculate the main direction of ground deformation $\left(\mathrm{V}_{\mathrm{r}}\right)$, so as to discriminate areas affected by subsidence or by landslides.

The methodology has been finally tested in a real scenario represented by an airfield runway located in Italy. The preliminary results have proven the viability of the method to evaluate the actual deformation pattern of the area, as demonstrated by way of comparison with the ground-truth survey.

\section{ACKNOWLEDGEMENTS}

The license for using the software ENVI SARscape ${ }^{\circledR}$ is granted by the ESA-approved project "MOBI: Monitoring Bridges and Infrastructure Networks" (EOhops proposal ID 52479, PI: Valerio Gagliardi). The Sentinel 1A SAR Products are (C) of the ESA (European Space Agency) delivered under the license to use.

\section{REFERENCES}

[1] A. Ferretti, C. Prati, F. Rocca, Permanent scatters in SAR interferometry. IEEE Trans Geosci Remote Sens 39(1):8-20, 2001, https ://doi.org/10.1109/36.898661

[2] A. Ferretti, C. Prati, F. Rocca Nonlinear subsidence rate estimation using permanent scatterers in differential SAR interferometry. IEEE Trans Geosci Remote Sens 38(5):2202-2212, 2000. https ://doi.org/10.1109/36.86887 8

[3] A. Ferretti, C. Prati, F. Rocca Analysis of permanent scatterers in SAR interferometry. 132:1-3, 2000. https ://doi.org/10.1109/igars s. 2000.861695 
[4] A. Ferretti, A.Fumagalli, F.Novali, C.Prati, F. Rocca, A.Rucci, A new algorithm for processing interferometric data-stacks: SqueeSAR ${ }^{\mathrm{TM}}$. IEEE Trans. Geosci. Remote Sens. 2011.

[5] R. Lanari, O.Mora, M. Manunta, J.J. Mallorqui, P. Berardino, E Sansosti, A small baseline approach for investigating deformation on full resolution differential SAR interferograms. IEEE Trans. Geosci. Remote Sens. 42, 1377-1386, 2004.

[6] P. Berardino, G. Fornaro, R. Lanari, E. Sansosti, A new algorithm for surface deformation monitoring based on small baseline differential SAR interferograms. IEEE Trans. Geosci. Remote Sens. 40, 2375 2383, 2003

[7] C. Colesanti, A. Ferretti, C. Prati et al., Monitoring landslides and tectonic motions with the Permanent Scatterers Technique. Eng Geol 68(1-2):3-14. https ://doi.org/10.1016/S0013 -7952(02)00195 -3,2003

[8] F. Koudogbo, A. Urdiroz, JG. Robles, G.Chapron, G.Lebon, V. Fluteaux, G. Priol Radar interferometry as an innovative solution for monitoring the construction of the Grand Paris Express metro network-first results. In: World tunnel conference, 2-25 April, Dubai, 2018

[9] F.D'Amico, V.Gagliardi, L.Bianchini Ciampoli, F.Tosti, Integration of InSAR and GPR Techniques for Monitoring Transition Areas in Railway Bridges. NDT\&E International. (in press) 2020.

[10] L. Bianchini Ciampoli, V. Gagliardi, A.Calvi, F. D’Amico, F. Tosti, Automatic network-level bridge monitoring by integration of InSAR and GIS catalogues. (2019) Proceedings of SPIE - The International Society for Optical Engineering, 11059, . DOI: 10.1117/12.2527299

[11] A. M. Alani, F. Tosti, L. Bianchini Ciampoli, V.Gagliardi, A Benedetto, Integration of GPR and InSAR methods for the health monitoring of masonry arch bridges. NDT\&E International. 2020 (in press).

[12] Z. Yang, F. Schmid, C. Roberts, Assessment of railway performance by monitoring land subsidence. In: 6th IET conference on railway condition monitoring (RCM 2014), pp 1-6. https ://doi. org/10.1049/cp.2014.1000, 2014.

[13] L. Bianchini Ciampoli, V. Gagliardi, C. Clementini, D. Latini, F. Del Frate, A. Benedetto, Transport Infrastructure Monitoring by InSAR and GPR Data Fusion, Surveys in Geophysics, 1-24, 2019.

[14] G. Barla et al., InSAR monitoring of tunnel induced ground movements. Geomechanik und Tunnelbau 9(1):15-22, 2016. https ://doi.org/10.1002/geot.20150 0052

[15] F. Tosti, V. Gagliardi, F. D'Amico, A.M. Alani, Transport infrastructure monitoring by data fusion 720 of GPR and SAR imagery information. Transp Res Proc 2020; 45:771-778. 721 https:// doi.org/10.1016/j.trpro.2020.02.097

[16] H.P. Sato, K.Abe, O. Ootaki, GPS-measured land subsidence in Ojiya City, Niigata Prefecture, Japan. Eng. Geol., 67, 379-390, 2003.

[17] A. Mossop, P. Segall, Subsidence at The Geysers Geothermal Field, N. California from a comparison of GPS and leveling surveys. Geophys. Res. Lett., 24, 1839-1842, 1997

[18] J. Olund, \& J. DeWolf, (2007). Passive Structural Health Monitoring of Connecticut's Bridge Infrastructure. Journal of Infrastructure Systems, 13(4), 330-339. doi:10.1061/(asce)10760342(2007)13:4(330

[19] P.Tang, D.Huber, \& B. Akinci, Characterization of Laser Scanners and Algorithms for Detecting Flatness Defects on Concrete Surfaces. Journal of Computing in Civil Engineering, 25(1), 31-42, 2011. doi:10.1061/(asce)cp.1943-5487.0000073

[20] D.Notti, G. Herrera, S. Bianchini, C. Meisina, J.C. García-Davalillo, F. Zucca, A methodology for improving landslide PSI data analysis. Int. J. Remote Sens. 2014, 35, 2186-2214

[21] A. Rosi, A.Agostini, V. Tofani, N. Casagli, A Procedure to Map Subsidence at the Regional Scale Using the Persistent Scatterer Interferometry (PSI) Technique. Remote Sens. 2014, 6, 10510-10522.

[22] R.Giraldo, P. Delicado, \& J. Mateu, Ordinary kriging for functionvalued spatial data. Environ Ecol Stat 18, 411-426, 2011. https://doi.org/10.1007/s10651-010-0143-y

[23] A.Papritz, A. Stein, "Spatial prediction by linear kriging". Spatial Statistics for Remote Sensing. Remote Sensing and Digital Image Processing. 1. p. 83. 2002. doi:10.1007/0-306-47647-9_6. ISBN 07923-5978-X.

[24] Sarmap. SARscape technical description, 2012. Internet resource accessed 2020-03-05 at:http://www.sarmap.ch/pdf/ /SARscapeTechnical.pdf 